\title{
Border Games in Cellular Networks
}

\author{
Márk Félegyházi*, Mario Čagalj ${ }^{\dagger}$, Diego Dufour*, and Jean-Pierre Hubaux* \\ * Laboratory for computer Communications and Applications (LCA) \\ EPFL, Switzerland \\ e-mail: \{mark.felegyhazi, diego.dufour, jean-pierre.hubaux\}@epfl.ch \\ ${ }^{\dagger}$ Faculty of Electrical Engineering, Mechanical Engineering and Naval Architecture (FESB), \\ University of Split, Croatia \\ e-mail: mario.cagalj@fesb.hr
}

\begin{abstract}
In each country today, cellular networks operate on carefully separated frequency bands. This separation is imposed by the regulators of the given country to avoid interference between these networks. But, the separation is only valid within the borders of a country, hence the operators are left on their own to resolve cross-border interference of their cellular networks. In this paper, we focus on the scenario of two operators, each located on one side of the border. We assume that they want to fine-tune the emitting power of the pilot signals (i.e., beacon signals) of their base stations. This operation is crucial, because the pilot signal power determines the number of users they can attract and hence the revenue they can obtain. In the case of no power costs, we show that there exists a motivation for the operators to be strategic, meaning to fine-tune the pilot signal powers of their base stations. In addition, we study Nash equilibrium conditions in an empirical model and investigate the efficiency of the Nash equilibria for different user densities. Finally, we modify our game model to take power costs into account. The game with power costs corresponds to the well-known Prisoner's Dilemma: The players are still motivated to adjust their pilot powers, but their strategic behavior leads to a sub-optimal Nash equilibrium.
\end{abstract}

Index Terms-Wireless networks, shared spectrum, pilot power control, cooperation, game theory, Nash equilibrium

\section{INTRODUCTION}

Today's cellular networks operate on separate frequency bands to avoid interference between them. The operators of these networks obtain an exclusive right to use a given frequency band in their respective country. However, the division based on frequency bands does not apply across national borders. The operators have to resolve their conflicts across the borders themselves. One of the issues is when mobile users of one operator attach to the network of the operator of the other country while still being in their own country. This problem is referred to as accidental roaming [15], [20]. The problem is significant, because there exist many examples of cities residing close to a national border such as Geneva, Basel or Aachen in Europe; San Diego and Detroit in the USA; or Hongkong and Singapore in Asia. Often, the operators make mutual agreements to resolve these problems, but these

The work presented in this paper was supported (in part) by the National Competence Center in Research on Mobile Information and Communication Systems (NCCR-MICS), a center supported by the Swiss National Science Foundation under grant number 5005-67322. agreements are difficult to enforce, because they require the mutual cooperation of the operators.

In this paper, we consider the problem of strategic behavior of operators on the border of their cellular networks. We consider $3 \mathrm{G}$ cellular networks, such as the Universal Mobile Telecommunication System (UMTS) for example, that are based on the Code Division Multiple Access (CDMA) technology [13], [27], [28]. Note however, that the problem we highlight in the paper applies to any CDMA network. In these networks, the base stations emit pilot signals to help users to assess the available channel quality and to attach to the base station with the best offered quality. According to the current definition in the UMTS standard, the pilot power for the base stations is determined at the network dimensioning phase and remains fixed afterward. However, as the number of users changes, the operators may adjust the network parameters. This slow adaptation of the pilot signal power is part of the network re-dimensioning process and hence it exists on a large time scale. On the other hand, the technology enables the base stations to quickly adapt their pilot signals to the actual usage. This fast adaptation technique is commonly referred to as cell breathing [13], [27], [28].

In this work, we assume that the operators want to adjust the power of the pilot signal of their base stations to attract more users over time. Several methods (e.g., cell-breathing [27], [28]) have been proposed to implement fast adaptation in CDMA networks. We survey them in Section II. In our paper, however, we focus on the slow adaptation problem. We study how the network operators can fine-tune their pilot power in the presence of other operators given a certain user distribution. We investigate whether this situation leads to a game and we study the properties of the equilibria of power control strategies.

The remainder of the paper is organized as follows. Section II surveys related work. In Section III, we present the system model and the corresponding game-theoretic concepts. In Section IV, we study whether the operators have an incentive to be strategic or not if there is no power cost. In Section V, we propose a distributed convergence algorithm to achieve the identified Nash equilibria. In Section VI, we extend the power control game to include the notion of power cost. We conclude in Section VII. 


\section{RELATED WORK}

Power control has been extensively studied in the context of cellular networking. Baccelli et al. [2] consider downlink power allocation and admission control in CDMA networks relying on stochastic geometry. Hanly and Tse [12] as well as Catrein et al. [4] consider power control and capacity in CDMA networks. But, there are only a few papers about pilot power optimization [18], [31].

Game theory is used to study the power control of user devices in wireless networks, notably in cellular systems as studied in [1], [11], [14], [17], [21], [23], [24], [32] and [34]. A general framework for resource allocation in wireless network is addressed in [5].

Recently, the coexistence of multiple Internet Service Providers (ISPs) was studied by Shakkottai and Srikant in [30]. They consider both transit and customer prices for the ISPs. They show that if the number of ISPs competing for the same customers is large, then it can lead to price wars. In another paper [29], Shakkotai et al. consider the problem of non-cooperative multi-homing in WLANs. Zemlianov and de Veciana [33] study a scenario in which users are able to choose between a cellular network and a Wi-Fi network. They show that congestion sensitive strategies are better than proximitybased strategies. Félegyházi and Hubaux [7] consider the competition between different operators in terms of pilot power control of their base stations. They show that in the pilot power control game a socially desirable Nash equilibrium exists and that it can be enforced by punishments.

\section{MODEL}

\section{A. System Model}

We consider a scenario with two cellular network operators $A$ and $B$. We assume that their networks are separated by a national border. The operators operate their network based on the principles of the CDMA method. We assume that the two operators acquired the same frequency band for their networks in their respective country. This means that their networks interfere along the border. We assume that each operator controls a set of base stations $(B S) \mathcal{B}_{i}$, where $i \in\{A, B\}$. We refer to the set of all base stations as $\mathcal{B}=\bigcup_{i} \mathcal{B}_{i}$. We also assume a set of users $\mathcal{M}$ equipped with wireless devices who access the communication network. For the sake of convenience, we assimilate the operators with their base stations and the users with their devices. In order to get an insight, we study the case in which each operator has one BS and we refer to the BS-s by the letters of their operators (i.e., base station $A$ and $B$ ). This single-cell model is often considered in the literature [16], [22]. The network scenario is shown in Figure 1.

We assume that the radios of the base stations and the mobile devices are compatible, meaning that any user is able to access the network via any of the base stations. We further assume that the antennas of the BS-s and wireless devices are omnidirectional. Note that the results derived in this paper are still valid if the operators use sectorized antennas that point towards the national border. Sectorized antennas have more impact in the general scenario, where the operators have

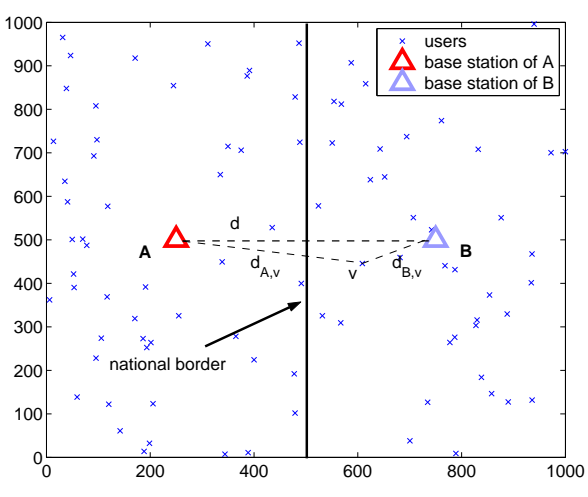

Fig. 1. Network scenario with two base stations.

several base stations each. The study of this general scenario is the main focus of our ongoing work.

Throughout this paper, we assume that the users are not associated with any of the operators (i.e., they are roaming users) and thus they attach to the base station with the best signal quality.

In CDMA networks, power control is used to mitigate the near-far effect [27], to optimize the transmission power of the devices and to reduce interference. In this paper, we focus on the downlink (or forward link) power control of the pilot signals emitted by the base stations. The pilot signal helps the wireless devices to perform the following tasks:

- detection of the available base stations,

- synchronization with them and

- estimation of the channel quality and handover decision based on this estimation.

In particular, we focus on the problem of how the network operators can determine the pilot signal power that will potentially attract the highest number of users. We leave the study of the competitive fast adaptation problem as a future work.

In the remainder of this section, we present the physical model of CDMA. As mentioned earlier, the pilot signal is used to attract users. If several users attach to a given base station, their transmissions are performed on different channels. In CDMA-based cellular networks, unlike GSM networks, channels are not separated in different frequencies, but use different codes. Hence each transmission uses the same frequency band. In theory, the codes from one base station are orthogonal, meaning that the transmissions to different receivers do not interfere with each other. In practice, there exists some interference between concurrent transmissions from a given base station because of multipath propagation. This interference is called the own-cell interference. In addition, there is an interference caused by the transmissions of other base stations, called the other-cell interference.

Let us consider the scenario shown in Figure 1. According to the physical model of signal propagation in a CDMA system [13], we can write the signal-to-interference-plus-noise ratio (SINR) of the pilot signal of base station $i \in\{A, B\}$ to user $v \in \mathcal{M}$ as: 


$$
\operatorname{SINR}_{i v}^{\text {pilot }}=\frac{G_{p}^{\text {pilot }} \cdot P_{i} \cdot d_{i v}^{-\alpha}}{N_{0} \cdot W+I_{o w n}^{\text {pilot }}+I_{\text {other }}^{\text {pilot }}}
$$

where $G_{p}^{p i l o t}$ is the processing gain for the pilot signal, $P_{i}$ is the power of the transmitted pilot signal of BS $i, d_{i v}$ is the distance between BS $i$ and user $v, \alpha$ is the path loss exponent, $N_{0}$ is the noise spectral density, $W$ is the available bandwidth, and $I_{\text {own }}^{\text {pilot }}$ as well as $I_{\text {other }}^{\text {pilot }}$ are the own-cell and the other-cell interferences that affect the pilot signal of BS $i$.

Let us first express the own-cell interference $I_{\text {own }}^{\text {pilot }}$ :

$$
I_{\text {own }}^{\text {pilot }}=\zeta \cdot d_{i v}^{-\alpha}\left(\sum_{w \in \mathcal{M}_{i}} T_{i w}\right)
$$

where $\zeta$ is the orthogonality factor (also called the owncell interference factor) that expresses the non-orthogonality between the different transmissions from BS $i$. Furthermore, $\mathcal{M}_{i}$ is the set of users at BS $i$ and $T_{i w}$ is the traffic power assigned to user $w \in \mathcal{M}_{i}$ by BS $i$.

Similarly, we can write the interference $I_{\text {other }}^{\text {pilot }}$ :

$$
I_{\text {other }}^{\text {pilot }}=\eta \cdot \sum_{j \neq i} d_{j v}^{-\alpha}\left(P_{j}+\sum_{w \in \mathcal{M}_{j}} T_{j w}\right)
$$

where $\eta$ is the other-to-own-cell interference factor, $d_{j v}$ is the distance between BS $j$ and user $v$. Furthermore $P_{j}$ is the pilot signal power of BS $j$, whereas $\mathcal{M}_{j}$ is the set of users at BS $j$ and $T_{j w}$ is the traffic power assigned to user $w \in \mathcal{M}_{j}$ by BS $j$.

Similarly to (1), we can express the SINR for the traffic signal $T_{i v}$ :

$$
\operatorname{SINR}_{i v}^{t r}=\frac{G_{p}^{t r} \cdot T_{i v} \cdot d_{i v}^{-\alpha}}{N_{0} \cdot W+I_{o w n}^{t r}+I_{o t h e r}^{t r}}
$$

where $G_{p}^{t r}$ is the processing gain for the traffic signal, $W$ is the available bandwidth, and $I_{\text {own }}^{t r}$ as well as $I_{\text {other }}^{t r}$ are the own-cell and the other-cell interferences that affect the traffic signal of BS $i$ to user $v$.

Let us write the own-cell interference $I_{\text {own }}^{t r}$ for the traffic signal as:

$$
I_{o w n}^{t r}=\zeta \cdot d_{i v}^{-\alpha}\left(P_{i}+\sum_{w \neq v, w \in \mathcal{M}_{i}} T_{i w}\right)
$$

and the interference from other BS-s $j$ as:

$$
I_{\text {other }}^{\text {tr }}=I_{\text {other }}^{\text {pilot }}=\eta \cdot \sum_{j \neq i} d_{j v}^{-\alpha}\left(P_{j}+\sum_{w \in \mathcal{M}_{j}} T_{j w}\right)
$$

Furthermore, we can express the carrier-to-interference ratio $(C I R)$ as a function of SINR:

$$
C I R_{i u}^{\text {pilot }}=\frac{\operatorname{SINR}_{\text {iv }}^{\text {pilot }}}{G_{p}^{\text {plot }}}
$$

Similarly, we can write the CIR of the traffic signal:

$$
C I R_{i v}^{t r}=\frac{S I N R_{i v}^{t r}}{G_{p}^{t r}}
$$

where $G_{p}^{t r}$ is the processing gain for the traffic signal from BS $i$ to user $v$.
In UMTS systems, the processing gain for the pilot signal is $G_{p}^{\text {pilot }}=256 \approx 14.3 \mathrm{~dB}$. The processing gain of the traffic signal $G_{p}^{t r}$ depends on the bitrate of the application running on the user device. In this paper, we refer to different types of communication as the traffic type, namely audio (12.2 kbps), video (144 kbps) and data (384 kbps) flows. ${ }^{1}$ Accordingly, we distinguish different requirements for different traffic types as presented in [13]. We summarize these parameters in Table I.

TABLE I

UMTS PARAMETERS (FROM [13], SECTION 8.2.1).

\begin{tabular}{c||c|c|c} 
traffic type & required SINR & processing gain & required CIR \\
\hline \hline pilot & $\approx-6 \mathrm{~dB}$ & $14.3 \mathrm{~dB}$ & $-20 \mathrm{~dB}$ \\
\hline audio, $12.2 \mathrm{kbps}$ & $5 \mathrm{~dB}$ & $25 \mathrm{~dB}$ & $-20 \mathrm{~dB}$ \\
\hline video, $144 \mathrm{kbps}$ & $1.5 \mathrm{~dB}$ & $14.3 \mathrm{~dB}$ & $-12.8 \mathrm{~dB}$ \\
\hline data, $384 \mathrm{kbps}$ & $1 \mathrm{~dB}$ & $10 \mathrm{~dB}$ & $-9 \mathrm{~dB}$
\end{tabular}

In wireless networks, the authorities impose a transmission power limit to the devices. In UMTS networks, the base stations must emit their signal below $43 \mathrm{dBm}=20 \mathrm{~W}$ [13]. This limit is called the downlink power budget. In addition, this power budget must be split between the control channel signals, such as the pilot signal, and the traffic channel transmissions. The actual utilization of the power budget is called the load of the base station. As the load increases, the bit-error-rate $(B E R)$ at the user devices increases exponentially [13]. Hence, the BS load is typically kept such that the BER does not exceed a certain threshold, for example $10^{-3}$. In this paper, we assume that the BS load is kept below $10 \mathrm{~W}$.

In order to determine the average usage of the two networks, we developed a numerical simulator in MATLAB. We summarize the parameters of our simulation in Table II. In each simulation run, we distribute the users according to the uniform distribution ${ }^{2}$ and calculate the number of users that attach to each of the BS-s based on the physical model developed in this section (i.e., using Equations (1)-(8) and the requirements shown in Table I). We repeat this experiment several times for each power setting and we obtain the average number of users at each BS.

\section{B. Game-theoretic Concepts}

We model competitive power control using game theory [6], [9], [10], [26]. Game theory is appropriate to model the strategic behavior of wireless devices or networks [3]. We define a two-player non-cooperative power control game $\mathbf{G}$ with the operators as players. In this game, the strategies of the operators determine the pilot transmission power of their base stations. Formally, we can write the strategy of operator $i$ as the pilot signal power value of his BS:

$$
s_{i}=P_{i}
$$

where $0 W<P_{i}<10 W$ is the pilot signal power of $\mathrm{BS}$ $i$. According to the UMTS standard, the BS-s transmit their pilot signal with approximately $33 \mathrm{dBm}=2 \mathrm{~W}$. We denote

\footnotetext{
${ }^{1}$ For simplicity, we consider only constant bitrate traffic.

${ }^{2}$ Note that we use a random uniform user distribution in our study, but our qualitative results hold for any user distribution.
} 
TABLE II

SIMULATION PARAMETERS (BASED ON [13]).

\begin{tabular}{l|l} 
Parameter & Value \\
\hline \hline simulation area size & $1 \mathrm{~km}^{2}$ \\
BS positions & $(250 \mathrm{~m}, 500 \mathrm{~m})$ and \\
& $(750 \mathrm{~m}, 500 \mathrm{~m})$ \\
default distance between BS-s, $d$ & $500 \mathrm{~m}$ \\
user distribution & random uniform \\
number of simulations & 500 \\
default path loss exponent, $\alpha$ & 4 \\
BS max power & $43 \mathrm{dBm}=20 \mathrm{~W}$ \\
BS max load & $40 \mathrm{dBm}=10 \mathrm{~W}$ \\
BS standard power, $P^{s}$ & $33 \mathrm{dBm}=2 \mathrm{~W}$ \\
BS min power & $20 \mathrm{dBm}=0.1 \mathrm{~W}$ \\
power control step size, $P_{\text {step }}$ & $0.1 \mathrm{~W}$ \\
orthogonality factor, $\zeta$ & 0.4 \\
other-to-own-cell interference factor, $\eta$ & 0.4 \\
user traffic types: & audio $(12.2 \mathrm{kbps})$ \\
& video $(144 \mathrm{kbps})$ \\
required CIR (audio, video, data): & data $(384 \mathrm{kbps})$ \\
expected incomes $\left(\theta_{\text {audio }}, \theta_{\text {video }}, \theta_{\text {data }}\right):$ & $-20 \mathrm{~dB},-12.8 \mathrm{~dB},-9 \mathrm{~dB}$ \\
\end{tabular}

this standard pilot power by $P^{s}$. We call the set of strategies of all players a strategy profile $s=\left\{s_{1}, s_{2}\right\} .{ }^{3}$ In our game, the players have the same strategy set $S$.

The operators define their strategies in order to maximize their expected payoff $u_{i}$ :

$$
u_{i}=\sum_{v \in \mathcal{M}_{i}} \theta_{v}
$$

where $\theta_{v}$ is the expected income obtained by serving user $v$ of a certain traffic type. Suppose that each user has the same traffic type, for example audio. Then the expected payoff obtained at $\mathrm{BS} i$ is:

$$
u_{i}=\left|\mathcal{M}_{i}\right| \cdot \theta_{\text {audio }}
$$

We further assume that the income ${ }^{4}$ per user increases according to the data rate of the given service, thus $\theta_{\text {audio }}<\theta_{\text {video }}<$ $\theta_{\text {data }}$. We obtain the expected income by performing several simulation runs with various pilot power settings as described in the previous section. This results in an expected payoff matrix for the two players. We apply the classic game-theoretic concepts on this payoff matrix. We express the payoffs of the players in Swiss francs $(\mathrm{CHF})$ to emphasize the monetary advantage.

In order to get an insight into the strategic behavior of the operators, we apply the following game-theoretic concepts. First, let us introduce the concept of best response. We can write $b r_{i}\left(s_{j}\right)$, the best response of player $i$ to the opponent's strategy $s_{j}$ as follows.

Definition 1: The best response of player $i$ to the profile of strategies $s_{j}$ is a strategy $s_{i}$ such that:

$$
b r_{i}\left(s_{j}\right)=\arg \max _{s_{i} \in S} u_{i}\left(s_{i}, s_{j}\right)
$$

\footnotetext{
${ }^{3}$ Note that one can easily extend the definitions in the power control game to several BS-s and operators.

${ }^{4}$ Note that the income is defined by the total amount of downloaded data, which can vary according to the length of communication sessions. If we change these income values, our results only change quantitatively, but not qualitatively.
}

One can see that if two strategies are mutual best responses to each other, then no player has a motivation to deviate from the given strategy profile. To identify such strategy profiles in general, Nash introduced the concept of Nash equilibrium in his seminal paper [25]. We can formally define the concept of Nash equilibrium (NE) as follows.

Definition 2: The pure-strategy profile $s^{*}$ constitutes a Nash equilibrium if, for each player $i$,

$$
u_{i}\left(s_{i}^{*}, s_{j}^{*}\right) \geq u_{i}\left(s_{i}, s_{j}^{*}\right), \forall s_{i} \in S
$$

where $s_{i}^{*}$ and $s_{j}^{*}$ are the Nash equilibrium strategies of player $i$ and $j$, respectively. In other words: In a Nash equilibrium, none of the players can unilaterally change his strategy to increase his payoff.

We use the concept of Pareto-optimality to characterize the efficiency of different strategy profiles.

Definition 3: The strategy profile $s$ is Pareto-optimal if there does not exist another strategy profile $s^{\prime}$ such that for each player $i$ :

$$
u_{i}\left(s_{i}^{\prime}, s_{j}^{\prime}\right) \geq u_{i}\left(s_{i}, s_{j}\right)
$$

with strict inequality for at least one player.

In other words, one cannot increase the payoff of a player without hurting the other player.

We present our results using a symmetric scenario of the base stations and assuming that the users are uniformly distributed in the simulation area. For other base station placements or user distributions, the Nash equilibrium strategies and payoffs are going to be asymmetric.

\section{Is There a Power Control Game?}

In this section, we study the behavior of the operators in a single-stage game. We first assume that one of the operators does not play and show that the other operator has an incentive to be strategic. ${ }^{5}$ Second, we consider the case in which both operators have the possibility to adjust their pilot power and show that they are better off by doing so. We obtain our simulation results using the simulation environment described in Section III-A.

\section{A. Only player $A$ is strategic}

First, we consider the case where only operator $A$ is strategic and adjusts the pilot power of his BS to attract more users, whereas operator $B$ operates his BS according to the standard pilot power of $P^{s}=2 \mathrm{~W}$. To quantify the advantage of the strategic player, we define the concept of normalized payoff difference $\Delta_{i}$.

Definition 4: The normalized payoff difference $\Delta_{i}$ is the normalized difference between the maximum payoff of player $i$ and his payoff using the standard power $P^{s}$ assuming that the other player $j$ uses $P^{s}$.

$$
\Delta_{i}=\frac{\max _{s_{i}}\left(u_{i}\left(s_{i}, P^{s}\right)\right)-u_{i}\left(P^{s}, P^{s}\right)}{u_{i}\left(P^{s}, P^{s}\right)}
$$

Suppose that there are on average 10 users of the data traffic type in the simulation area. We show the payoffs of players

\footnotetext{
${ }^{5}$ Due to symmetry, we only show the results for player $A$.
} 


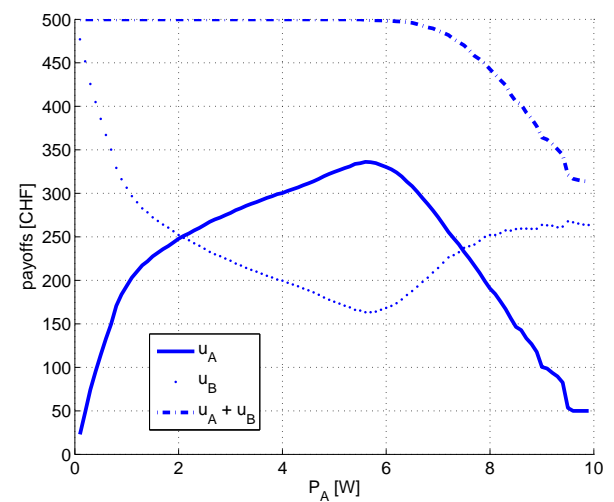

(a)

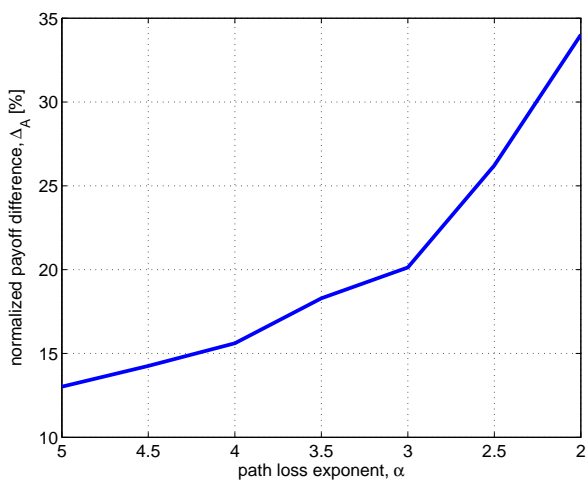

(c)

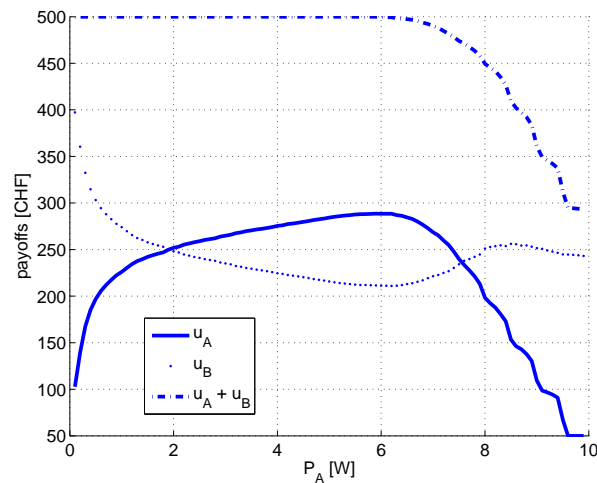

(b)

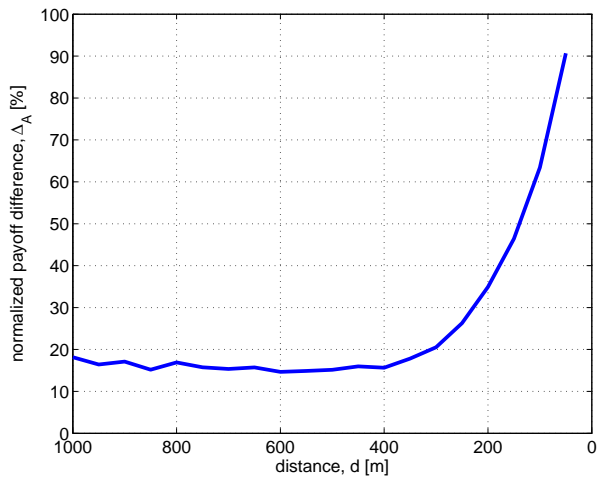

(d)

Fig. 2. Payoffs of the players as a function of the pilot power of player $A$ : (a) for $\alpha=2$ and (b) for $\alpha=4$. We also show the normalized payoff difference $\Delta_{A}$ as a function of (c) the path loss exponent $\alpha$ and (d) the distance $d$ between the two BS-s.

$A$ and $B$ as a function of the pilot signal power $P_{A}$ as well as the sum of their payoffs in Figure 2. Figure 2 a shows these payoffs for $\alpha=2$, whereas Figure 2b presents the same results for $\alpha=4$. We observe that in both cases the operators are able to serve all users in the area using certain power values. If all users are served, then the game is a zero-sum game. In the zero-sum game, if player $A$ adjusts his pilot power and obtains the increase of $\Delta_{A}$, he causes the decrease of $\Delta_{A}$ in the payoff of the non-strategic player $B$. Furthermore, the payoff function of operator $A$ has a unique maximum point. It is interesting to observe that the maximum payoff point requires a higher pilot power than $P^{s}=2 W$. Hence, we conclude that operator $A$ has an incentive to adjust his pilot signal. Note that we obtain qualitatively the same result for different user traffic types.

Figures $2 \mathrm{a}$ and Figure $2 \mathrm{~b}$ show that the value of the normalized payoff difference $\Delta_{A}$ depends on the parameter $\alpha$. We show this dependency in Figure 2c. One can observe that $\Delta_{A}$ increase as $\alpha$ decreases. The reason is that by low $\alpha$ values the pilot signals propagate more easily giving a higher gain to $A$ if he uses higher pilot power. The value of $\Delta_{A}$ also depends on the distance $d$ between the two BS-s as shown in Figure $2 \mathrm{~d}$. As the distance decreases, $\Delta_{A}$ increases exponentially. The reason for this increase is the same as discussed before. In the remainder of the paper, we choose the conservative default values $\alpha=4$ and $d=500 m$ for the simulations. We will show that even with these conservative values, the players have an incentive to fine-tune their pilot powers.

\section{B. Both operators are strategic}

In the second set of simulations, we assume that both operators adjust their pilot power. We still consider 10 data users in the simulation area. We provide the payoff of player $A$ as a function of his pilot power $P_{A}$ in Figure $3 \mathrm{a}$. We obtain different payoff curves as the pilot power of the other BS $P_{B}$ increases. We can observe that each of the payoff functions has a unique maximum point for $P_{A}$. Moreover, this maximum point depends on the pilot power of the other BS, $P_{B}$. For low values of $P_{B}$, the maximum payoff value decreases as $P_{B}$ increases. In Figure $3 \mathrm{~b}$, we show the payoff surface for operator $A$ as a function of the pilot power values of the two BS-s.

Using the two payoff surfaces, we derive the best response functions (i.e., the set of maximum payoff points) for the operators as shown in Figure 4 for two different user densities. Based on the concept of best responses introduced in Section III-B, we can identify the Nash equilibria in the power control game as shown in Figures $4 \mathrm{a}$ for 10 data users and Figures $4 \mathrm{~b}$ for 100 data users. We see that there exists a unique Nash equilibrium point defined as the crossing point of the two best response functions. Note that for 10 data users the Nash equilibrium strategy profile defines $P_{A}=P_{B}=6 \mathrm{~W}$, which are higher than the standard pilot powers. For 100 data users 


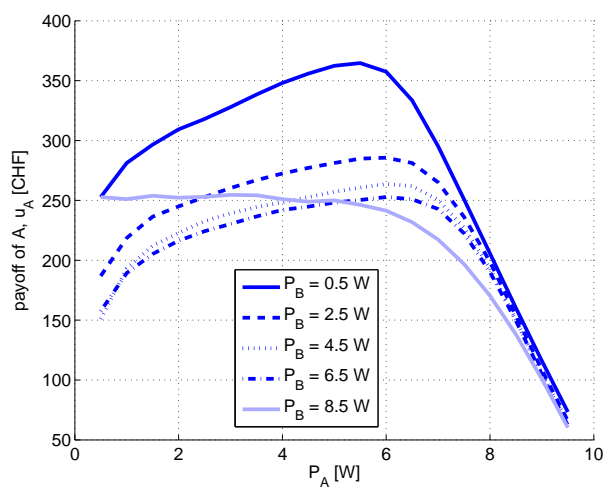

(a)

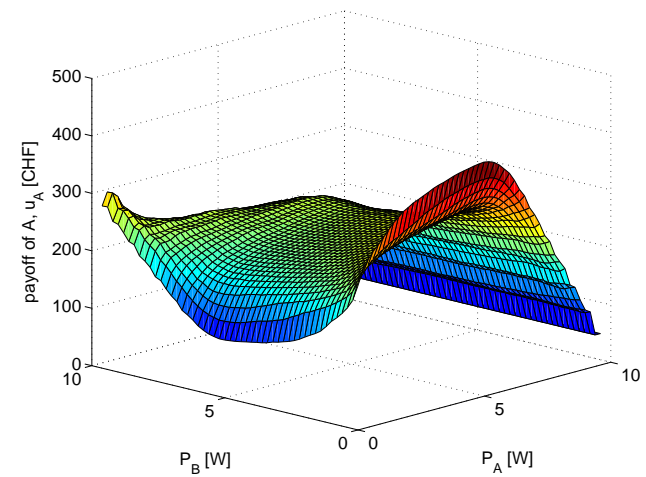

(b)

Fig. 3. Payoff of player $A$ as a function of his pilot power. Both operators are strategic, hence we present this payoff for various values of $P_{B}$ in (a). We show the complete payoff surface in (b).

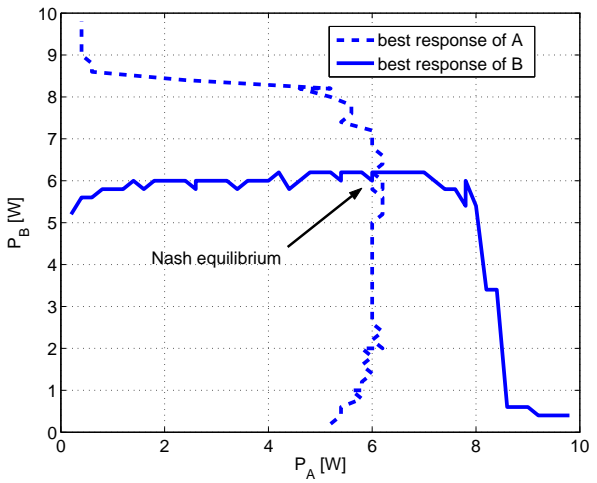

(a)

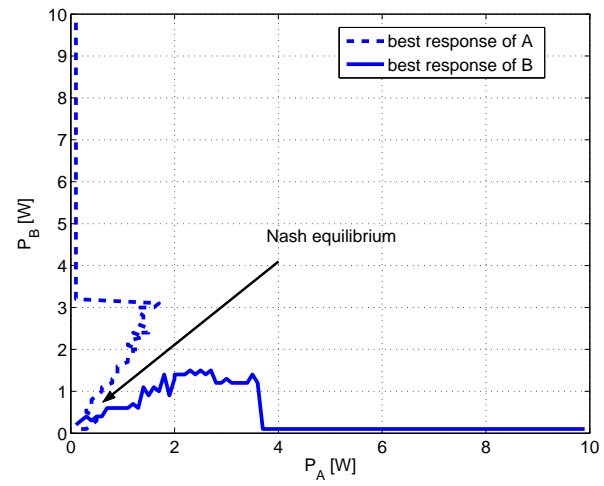

(b)

Fig. 4. Best response functions for the two players with (a) 10 data users, (b) 100 data users.

the Nash equilibrium strategy profile defines $P_{A}=P_{B}=$ $0.5 \mathrm{~W}$. The reason is that the capacities BS-s saturate by using a relatively small power and hence there is no motivation for them to go above these pilot power values.

Next, we study the pilot power values in the Nash equilibrium as a function of the number of users. We show the results in Figure 5. Due to symmetry in the user distributions, the Nash equilibrium pilot power is the same for both players. We observe that the Nash equilibrium pilot powers decrease as the number of users increases. For high user densities, the Nash equilibrium pilot powers stabilize at the value of $0.5 \mathrm{~W}$.

In the following set of experiments, we study the efficiency of the system in a Nash equilibrium with respect to the case in which the players both use the standard power $P^{s}$. To this end, we investigate the payoff region, i.e. the payoff values for various pilot power levels. We identify the payoffs corresponding to the Nash equilibrium, the standard pilot power setting using $P^{s}$ and the payoffs that correspond to Pareto-optimal strategy profiles. In particular, we can define the Pareto boundary as the set of Pareto-optimal payoff points. In our case, the Pareto-optimal payoff points characterize the system-efficient solutions.

Figure $6 \mathrm{a}$ shows the achieved payoffs as a function of the pilot power values $P_{A}$ and $P_{B}$ for 10 data users. We

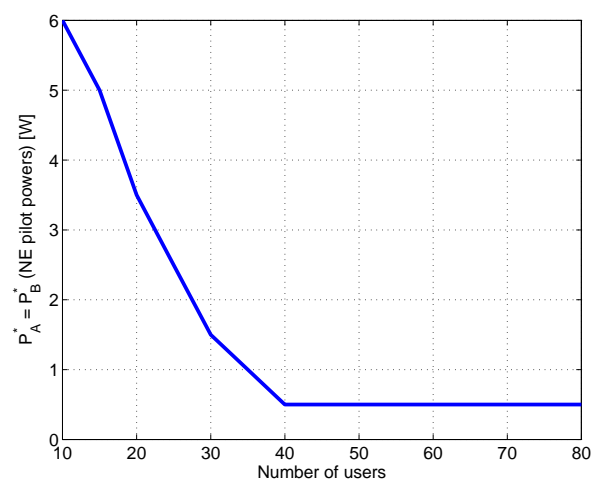

Fig. 5. Nash equilibrium pilot power values as a function of the user density.

observe that in this case the Pareto boundary defines a straight line, because in a Pareto-optimal strategy profile each user in the system is attached to one of the BS-s. Furthermore, the standard pilot powers and the Nash equilibrium strategy profile result in the same payoffs for the players and in addition they both lie on the Pareto boundary. This means that the players achieve a desirable state from the system point of view. Recall, however, that in this case the Nash equilibrium strategy profile 


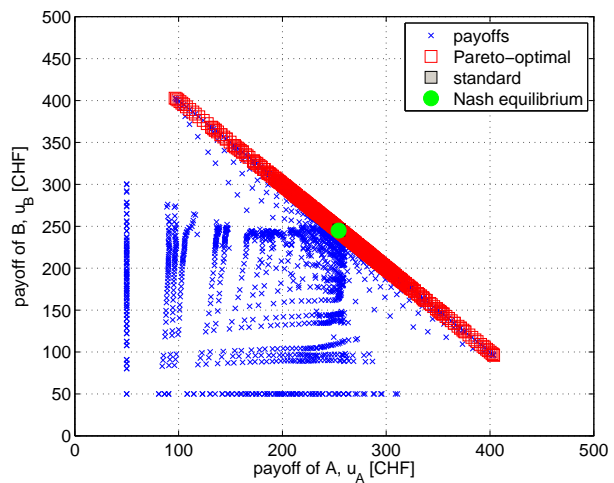

(a)

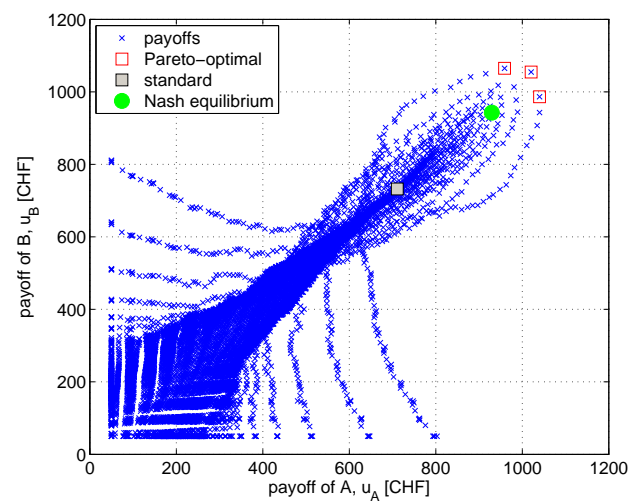

(b)

Fig. 6. The payoff region with all possible payoffs for (a) 10 data users and (b) 100 data users. We highlight the Nash equilibrium, the payoff of the standard powers and all Pareto-optimal points.

requires higher pilot powers than the standard setting.

We present the payoffs for 100 data users in Figure $6 \mathrm{~b}$. In this case the Pareto-optimal points do not form a straight line anymore, because some users cannot be served. Another observation is that the Nash equilibrium is still close to Paretooptimality, but the standard solution becomes very inefficient.

Following the previous experiment, we formally express the efficiency of the standard and the Nash equilibrium solutions compared to the best Pareto-optimal point (i.e., the Paretooptimal strategy profile in which the sum of the payoffs for the two players is maximized). To this end, let us define the following two concepts:

Definition 5: The price of anarchy [19] is the ratio between the total payoff achieved by the two players in the best Paretooptimal point and in the Nash equilibrium.

Definition 6: The price of conformance is the ratio between the total payoff achieved by the two players in the best Paretooptimal point and when using the standard pilot powers $P^{s}$ (i.e., being non-strategic).

We perform a set of experiments to measure these values for increasing user densities. Figure 7 presents the price of anarchy and the price of conformance as a function of the user density assuming they have data traffic. We see that both prices increase as the number of users increases. As we have seen in Figure 6a, both the standard payoff point and the Nash equilibrium achieve Pareto-optimality if there is a small number of users. Hence, the two prices are very close to one. As the user density increases, we observe that both prices increase and then stabilize around a constant value. Note, however, that the price of anarchy stabilizes close to one, whereas the price of conformance stabilizes around 1.4. This shows that for a high number of users, the players can achieve a higher payoff if both of them are strategic.

\section{Convergence to a Nash Equilibrium}

We have seen in the previous section that the expected payoff function for a certain player is continuous and has a unique maximum point. In this section, we propose a distributed algorithm to achieve the Nash equilibrium in a

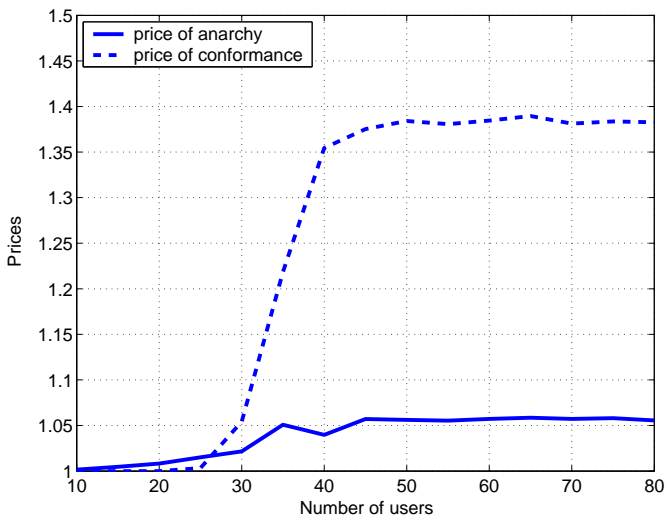

Fig. 7. The price of anarchy and the price of conformance as a function of the user density.

given scenario. The algorithm is similar to the better-response dynamics [8], i.e., where each player tries to improve his payoff in each step. We provide the pseudo-code as shown in Algorithm 1.

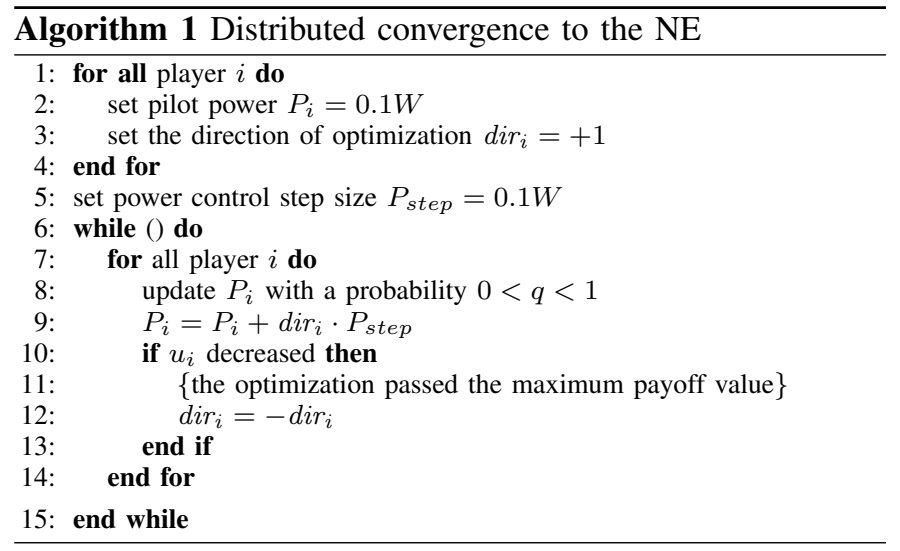

Figure 8a shows the evolution of the pilot power values applying Algorithm 1. We observe that the pilot power values follow the linear increase defined in the algorithm. After reaching the Nash equilibrium pilot power values, the algorithm 


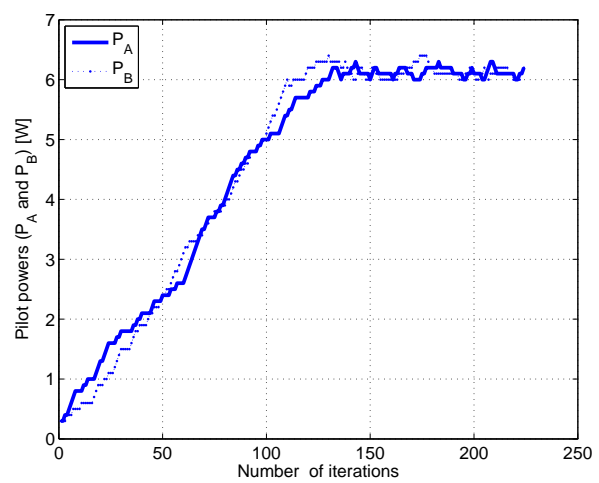

(a)

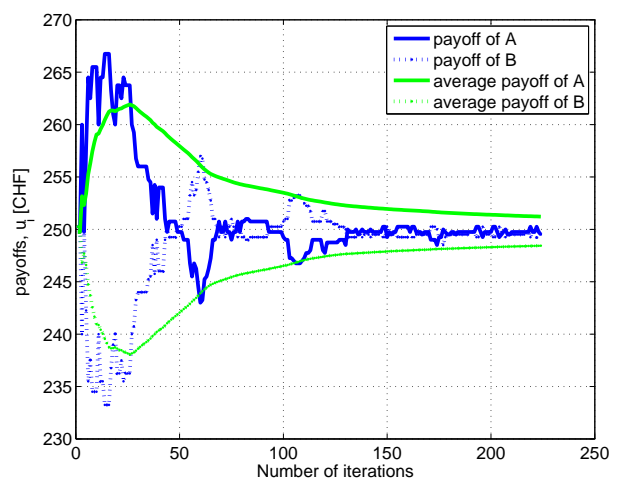

(b)

Fig. 8. Convergence to the Nash equilibrium using Algorithm 1. We present (a) the evolution of the pilot power values and (b) evolution of the payoffs.

stabilizes after certain steps.

Figure $8 \mathrm{~b}$ shows the evolution of the payoffs during the convergence process. We see that the algorithm deviates from the Nash equilibrium payoffs while the pilot powers increase. As soon as the pilot powers reach the Nash equilibrium strategies, the payoffs remain close to the Nash equilibrium payoffs as well.

\section{Power Control Game with Power Cost}

We have seen that the operators are able to serve all users in the area if the user density is low. We observe, however, that the Nash equilibrium pilot powers are higher than the standard value. Recall that the payoff function defined in (10) does not include the possible cost due to the operation with high pilot power. Let us now extend the expected payoff function defined in (10) to capture this important aspect of the power control game. We introduce two cost values for each player. The first cost denoted by $C_{i}^{o p}$ shows the operating cost of a BS $i$. This includes the aging of devices and hence the maintenance costs. The other cost, $C_{i}^{s u b j}$, expresses the subjective cost of player $i$. This covers every other aspect such as the risk of lawsuits or potential bad reputation due to high emission power. Without loss of generality, we assume that these costs are an increasing function of the downlink transmission power of the base stations.

According to the above description, we can extend the notion of expected payoff as:

$$
u_{i}=\left(\sum_{v \in \mathcal{M}_{i}} \theta_{v}\right)-C_{i}^{o p}-C_{i}^{s u b j}
$$

We define a non-cooperative power control game with the new expected payoff function introduced in (16) and denote it by $\hat{\mathbf{G}}$. We assume that the players are able to calculate the Nash equilibrium of the original game $G$. We define the strategy in the extended game $\hat{\mathbf{G}}$ as the choice between the standard and the Nash equilibrium strategies. Formally, we can write the strategies in $\hat{\mathbf{G}}$ as:

$$
s_{i}=\left\{P_{i}^{*}, P^{s}\right\}
$$

Let us call $U$ the expected payoff that the players obtain by serving half of the total number of users. As we have seen in
Section IV-B, if they play the Nash equilibrium strategy profile by low user densities, then it requires a higher pilot power from each operator. Without loss of generality, we denote by $C^{*}$ the additional cost imposed by the Nash equilibrium compared to the standard pilot power setting $P^{s}$. The cost $C^{*}$ includes both the operating and the subjective costs. Recall that we defined the normalized payoff difference $\Delta_{A}$ in Section IV-A. Due to the symmetry $\Delta_{A}=\Delta_{B}$ and we denote it by $\Delta$. In the extended game $\hat{\mathbf{G}}$, we assume that the normalized payoff difference is higher than the corresponding cost of using higher pilot power, thus $\Delta>C^{*}$.

We present the payoff matrix of the game $\hat{\mathbf{G}}$ in Table III. In each payoff pair, the first payoff belongs to player $A$, whereas the second to player $B$.

TABLE III

PAYOFF MATRIX OF THE GAME $\hat{\mathbf{G}}$.

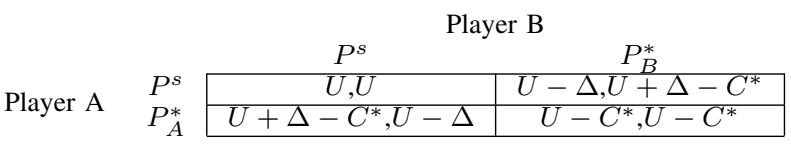

To emphasize the structure of the payoff matrix, let us substitute the values $U=3, \Delta=2$ and $C^{*}=1$. Substituting these values in Table III, we obtain Table IV. From the payoff matrix, one can realize that the game $\hat{\mathbf{G}}$ is equivalent to the well-known Prisoner's Dilemma [9], [10], [26]. Analogously, the strategy $P^{s}$ corresponds to cooperation, whereas the strategy $P_{i}^{*}$ corresponds to defection. This means that in the Nash equilibrium, each player uses high power and the resulting payoffs are lower than if both had complied.

\section{TABLE IV}

THE EXTENDED POWER CONTROL GAME $\hat{\mathbf{G}}$ CORRESPONDS TO THE PRISONER'S DILEMMA.

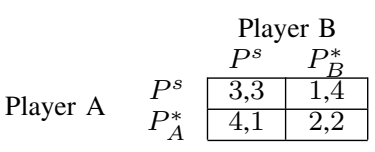




\section{CONCLUSION}

In this paper, we have studied the problem of competitive pilot power control in two CDMA networks that reside on the two sides of a national border. We have investigated whether the operators of these networks have an incentive to adjust their pilot signal powers or not. To get an insight into the problem, we have considered the single cell-case with two base stations. Initially, we have assumed that only one operator can adjust the pilot signal power of his base station. We have shown that he has an incentive to be strategic and quantified the effect of various parameters on the increase of his payoff. We have shown that if both operators are strategic and the user density is low, then being strategic or not results in similar payoffs. We have recognized that the two solutions require different pilot powers. If the user density is high, then the Nash equilibrium is more efficient than using the standard pilot powers, which suggests that the operators again have an incentive to be strategic. Finally, we have extended the payoff function to include the cost of using high pilot powers. We have established the analogy between the power control game with power cost in case of low user densities and the wellknown Prisoner's Dilemma.

In terms of future work, we will extend the study of the single cell case to scenarios including several base stations. Because this case is fairly complex, we will propose distributed algorithms to identify and achieve Nash equilibria. Furthermore, we will consider power control games for scenarios, where users are associated with one of the operators. Finally, we will study the enforcement of desirable power signal levels through power pricing.

\section{REFERENCES}

[1] T. Alpcan, X. Fan, T. Basar, M. Arcak, and T. J. Wen. Power control for multicell CDMA wireless networks: A team optimization approach. In Proc. of WiOpt'05, Apr. 2005.

[2] F. Baccelli, B. Błaszczyszyn, and F. Tournois. Downlink admission/congestion control and maximal load in CDMA networks. In Proceedings of the IEEE Conference on Computer Communications (INFOCOM '03), March 30 - April 32003.

[3] L. Buttyán and J.-P. Hubaux. Security and Cooperation in Wireless Networks. Cambridge University Press, to appear, 2007.

[4] D. Catrein, L. A. Imhof, and R. Mathar. Power control, capacity, and duality of uplink and downlink in cellular CDMA systems. IEEE Transactions on Communications, 52(10):1777-1785, Oct. 2004.

[5] M. Dramitinos, C. Courcoubetis, and G. D. Stamoulis. Auction-based resource reservation in $2.5 / 3 \mathrm{G}$ networks. Kluwer/ACM Mobile Networks and Applications (MONET), 2003.

[6] M. Félegyházi and J.-P. Hubaux. Game theory in wireless networks: A tutorial. Technical Report LCA-REPORT-2006-002, EPFL, Feb. 2006.

[7] M. Félegyházi and J.-P. Hubaux. Wireless operators in a shared spectrum. In Proceedings of the IEEE Conference on Computer Communications (INFOCOM '06), April 23-29 2006.

[8] J. W. Friedman and C. Mezzetti. Learning in games by random sampling. Journal of Economic Theory, 98:55-84, 2001.

[9] D. Fudenberg and J. Tirole. Game Theory. MIT Press, 1991.

[10] R. Gibbons. A Primer in Game Theory. Prentice Hall, 1992.

[11] D. Goodman and N. Mandayam. Network assisted power control for wireless data. Mobile Networks and Applications (MONET), 6:409-415, 2001.

[12] S. V. Hanly and D. N. Tse. Power control and capacity of spread spectrum wireless networks. Automatica, 35(12):1987-2012, Dec. 1999.

[13] H. Holma and A. Toskala, editors. WCDMA for UMTS. John Wiley \& Sons, Inc., New York, NY, USA, 2002.

[14] J. Huang, R. Berry, and M. Honig. Auction-based spectrum sharing. ACM/Kluwer Journal of Mobile Networks and Applications (MONET) special issue on WiOpt'04, 11:405-418, 2006.
[15] INTUG. International mobile roaming. An INTUG response to the DG Information Society Second Phase Consultation on Roaming Charges April 2006, May 2006.

[16] W. C. Jakes, editor. Microwave Mobile Communications. John Wiley \& Sons, Inc. - IEEE Press, 1994.

[17] H. Ji and C.-Y. Huang. Non-cooperative uplink power control in cellular radio systems. Wireless Networks (WINET), 46(3):233-240, 1998.

[18] D. Kim, Y. Chang, and J. W. Lee. Pilot power control and service coverage support in CDMA mobile systems. In Proceedings of IEEE Vehicular Technology Conference (VTC'99), pages 1464-1468, 1999.

[19] E. Koutsoupias and C. Papadimitriou. Worst-case equilibria. In Proceedings of the 16th Annual Symposium on Theoretical Aspects of Computer Science (STACS'99), March 1999.

[20] A. Lee. Optimizing traffic management. Starhome $\mathrm{GmbH}$, (http://www.starhome.com), 2006.

[21] J. W. Lee, R. R. Mazumdar, and Ness B. Shroff. Downlink power allocation for multi-class CDMA wireless networks. In Proceedings of the IEEE Conference on Computer Communications (INFOCOM '02), June 23-27 2002.

[22] P. Liu, P. Zhang, S. Jordan, and M. L. Honig. Single-cell forward link power allocation using pricing in wireless networks. IEEE Transactions on Wireless Communications, 3(2):533-543, March 2004.

[23] A. B. MacKenzie and S. B. Wicker. Game theory and the design of self-configuring, adaptive wireless networks. IEEE Communications Magazine, Nov. 2001.

[24] F. Meshkati, M. Chiang, H. V. Poor, and S. Schwartz. A non-cooperative power control game for multi-carrier CDMA systems. In Proc. IEEE Wireless Communications and Networking Conference (WCNC), March 2005.

[25] J. Nash. Non-cooperative games. The Annals of Mathematics, 54(2):286-295, 1951

[26] M. J. Osborne and A. Rubinstein. A Course in Game Theory. The MIT Press, Cambridge, MA, 1994.

[27] T. S. Rappaport. Wireless Communications: Principles and Practice (2nd Edition). Prentice Hall, 2002.

[28] M. Schwartz. Mobile Wireless Communications. Cambridge Univ. Press, 2005.

[29] S. Shakkottai, E. Altman, and A. Kumar. The case for non-cooperative multihoming of users to access points in IEEE 802.11 WLANs. In Proceedings of the IEEE Conference on Computer Communications (INFOCOM 'O6), April 23-29 2006.

[30] S. Shakkottai and R. Srikant. Economics of network pricing with multiple ISPs. In Proceedings of the IEEE Conference on Computer Communications (INFOCOM '05), March 13-17 2005.

[31] P. Värbrand and D. Yuan. A mathematical programming approach for pilot power optimization in WCDMA networks. In Proceedings of the Australian Telecommunications, Networks and Applications Conference (ATNAC '03), December 2003.

[32] M. Xiao, N. B. Schroff, and E. K. P. Chong. A utility-based power control scheme in wireless cellular systems. IEEE/ACM Trans. on Networking, 11(10):210-221, March 2003.

[33] A. Zemlianov and G. de Veciana. Cooperation and decision making in wireless multi-provider setting. In Proceedings of the IEEE Conference on Computer Communications (INFOCOM '05), March 13-17 2005.

[34] C. Zhou, P. Zhang, M. L. Honig, and S. Jordan. Two-cell power allocation for downlink CDMA. IEEE Transactions on Wireless Communications, 3(6):2256-2266, Nov. 2004. 